\title{
A ocorrência de ceceio em crianças de oito a 11 anos em escolas municipais
}

\author{
The occurrence of lisp in eight- to 11-year-old children \\ from municipal schools
}

\author{
Vanessa Roses Monteiro ${ }^{1}$, Silvana Maria Brescovici², Susana Elena Delgado ${ }^{3}$
}

\begin{abstract}
RESUMO
Objetivos: Verificar a ocorrência de ceceio em crianças de oito a 11 anos das escolas municipais de Itaqui/RS, de acordo com sexo e faixa etária, além de verificar a associação do ceceio com possíveis fatores de risco, tais como hábitos de sucção (chupeta, dedo e mamadeira) até os seis anos de idade, má oclusão dentária, respiração oral e praxias de língua. Métodos: Foi realizado um estudo observacional, exploratório, contemporâneo, prospectivo e de grupo, por meio de questionário com os responsáveis e de avaliação fonoaudiológica das estruturas, das praxias da língua, da presença de sinais sugestivos de respiração oral e articulação dos sons da fala. Utilizou-se estatística não-paramétrica (Qui-quadrado) para verificar associação entre as variáveis. Resultados: Participaram 200 crianças (50\% meninos e 50\% meninas) de oito a 11 anos. A frequência de ceceio foi de 19\%, maior nas meninas e observouse que ceceio anterior diminui com o aumento da idade o que não ocorreu com o ceceio lateral. Os sinais sugestivos de respiração oral $(\mathrm{p}=0,037)$, o uso prolongado da mamadeira $(\mathrm{p}=0,027)$, as alterações de praxias de língua ( $\mathrm{p}=0,002)$, e as alterações de oclusão $(\mathrm{p}=0,0001)$, foram os fatores de risco que mostraram associação estatística. Conclusões: Foi alta a frequência de ceceio e maior no sexo feminino. A maioria dos fatores de risco associou-se significativamente.
\end{abstract}

Descritores: Patologia da fala e linguagem; Transtornos da articulação; Comportamento de sucção; Oclusão dentária; Respiração bucal

\section{INTRODUÇÃO}

Os distúrbios articulatórios de fala são os distúrbios de comunicação mais prevalentes em escolares e pré-escolares. Há referências do National Institute on Deafness and Other Communication Disorders (NIDCD) que 10\% da população apresentam distúrbio articulatório de fala e para $80 \%$ destas crianças, tal distúrbio é suficientemente grave $\mathrm{e}^{(1)}$.

A produção do $/ \mathrm{s} /$, conforme descrito em estudo de medidas dos atributos acústicos da fricativa /s/, exige que a borda de língua assuma forma e posição específicas e extremamente precisas. A produção correta desse fonema é caracterizada pelo

Trabalho realizado na Universidade Luterana do Brasil - ULBRA - Porto Alegre (RS), Brasil.

(1) Fonoaudióloga clínica - Porto Alegre (RS), Brasil; Especialização em Motricidade Oral pela Universidade Luterana do Brasil - ULBRA - Porto Alegre (RS), Brasil.

(2) Mestre, Professora Adjunto do Curso de Fonoaudiologia da Universidade Luterana do Brasil - ULBRA - Porto Alegre (RS), Brasil.

(3) Mestre, Professora Adjunto do Curso de Fonoaudiologia da Universidade Luterana do Brasil - ULBRA - Porto Alegre (RS), Brasil.

Endereço para correspondência: Silvana Maria Brescovici. R. Barão de Ubá, 651, Bela Vista, Porto Alegre - RS, CEP: 90450-090. E-mail: silvana@brescovici.com.br

Recebido em: 26/8/2008; Aceito em: 4/11/2008 posicionamento da ponta da língua na região alveolar e por um formato adequado da borda. Além disso, a mandíbula deverá estar corretamente posicionada para direcionar o fluxo aéreo contra os incisivos inferiores ${ }^{(2)}$. Quando estas condições são modificadas o som é produzido de forma incorreta ${ }^{(3)}$, podendo estar distorcido ${ }^{(4)}$.

Modificações das estruturas e ou espaço intra-oral podem alterar os pontos articulatórios, levando a distorções principalmente dos fonemas fricativos ${ }^{(5)}$, que por serem contínuos são os sons mais fáceis de serem distorcidos ${ }^{(4)}$. Assim, alterações funcionais e estruturais dos órgãos fonoarticulatórios podem ser a causa de tal desvio fonético ${ }^{(5)}$.

Na literatura, há divergência de terminologia para referir-se à distorção dos fonemas fricativos alveolares /s/. De acordo com o Comitê de Motricidade Orofacial da Sociedade Brasileira de Fonoaudiologia ${ }^{(6)}$, o desvio fonético do tipo ceceio anterior ou lateral é o som do fonema/s/, uma fricativa alveolar sendo pronunciado como interdental, com apoio da ponta da língua nos dentes. Já, sigmatismo, segundo o Comitê, é o emprego frequente ou a repetição viciosa dos sibilantes. No presente estudo será adotado o termo ceceio, assim como, nas citações se respeitará a terminologia utilizada originalmente pelos autores.

O ceceio é a ocorrência mais frequentemente encontrada 
dentro das alterações fonéticas, sendo que aproximadamente 90\% de todos os falantes com distúrbios articulatórios apresentam dificuldades com esse som $^{(7)}$.

Estudos que investigaram associação do ceceio anterior com a idade, gênero, tipo de mordida, deglutição atípica, oclusão dentária, tônus muscular rebaixado, presença de hábitos orais, modo respiratório, presença de diastema, crescimento crânio facial mostraram resultados divergentes ${ }^{(3-5,8-13)}$. Porém, a maioria deles mostra que o sigmatismo diminui com o aumento da idade ${ }^{(4,8-9,11-14)}$. Entretanto, são poucos os estudos realizados em crianças com mais de sete anos ${ }^{(10,13-14)}$. Da mesma forma, são poucos os que investigaram o ceceio lateral ${ }^{(13)}$.

No posto de Saúde/SUS em Itaqui/RS, o serviço de Fonoaudiologia recebe frequentemente encaminhamentos de crianças, oriundas da rede escolar municipal com queixas relacionadas à fala. Evidencia-se com frequência a distorção sonora do fonema $/ \mathrm{s} /$.

Este estudo teve por objetivos: verificar a ocorrência de ceceio em crianças de oito a 11 anos, nas escolas municipais de Itaqui/RS. Além disso, mais especificamente verificar a ocorrência de ceceio conforme gênero e faixa etária; analisar a associação com possíveis fatores de risco tais como tipo de mordida, respiração oral e praxias de língua (estalar, fora/ dentro, afinar/alargar, canolar ponta, canolar meio, sugar, cima/baixo).

Por meio dos resultados pretende-se obter referências teóricas voltadas à caracterização de desvio fonético do tipo ceceio e, a partir delas, promover ações preventivas no âmbito escolar, auxiliando no diagnóstico precoce para possíveis encaminhamentos e intervenções terapêuticas.

\section{MÉTODOS}

Este estudo de delineamento transversal, observacional, prospectivo, de grupo, pesquisou crianças entre oito e 11 anos, de ambos os sexos que frequentavam a rede escolar municipal de Itaqui.

Foram avaliadas 200 crianças distribuídas nos seguintes grupos etários:

- oito anos e um mês a oito anos e 11 meses ( 25 meninos e 25 meninas);

- nove anos e um mês a nove anos e 11 meses (25 meninos e 25 meninas);

- dez anos e um mês a dez anos e 11 meses (25 meninos e 25 meninas);

- 11 anos e um mês a 11 anos e 11 meses ( 25 meninos e 25 meninas).

Para que a pesquisa tivesse uma amostra adequadamente homogênea, foram considerados como fatores de exclusão crianças que estivessem fazendo ou que já tivessem realizado tratamento fonoaudiológico e/ou ortodôntico, crianças com alterações neurológicas, psiquiátricas, deficiência auditiva e visual.

A presente pesquisa foi autorizada pela Secretaria Municipal de Educação e Cultura de Itaqui, e aprovada pelo Comitê de Ética em Pesquisa da Universidade Luterana do Brasil com o protocolo $\mathrm{n}^{\circ} 342 \mathrm{H} / 2005$.

Encaminhou-se o Termo de Consentimento Livre e Es- clarecido (TCLE) e questionário para pais e responsáveis de 300 crianças (Anexo 1), tendo retornado apenas 200 questionários.

O questionário teve por objetivo identificar fatores de exclusão e possíveis fatores de risco tais como hábitos de sucção não-nutritivos (chupeta e dedo) e nutritivos (mamadeira) até os seis anos de idade.

A identificação das alterações da fala foi realizada pela análise perceptivo-auditiva da examinadora e da inspeção visual do movimento de língua, durante a avaliação da fala espontânea (conversa informal), repetição de pequenas frases (O sapo pulou na moça; Selma pintou com pincel; A polícia tocou a sirene; Sônia assoprou a vela; Sueli ficou assustada); e nomeação de figuras com vocábulos com /s/ em posição inicial e não-inicial (sapato, bolsa, cenoura, travesseiro, circo, piscina, sorvete, palhaço, super-homem, açucareiro). Considerou-se alteração da fala presente, quando ela ocorreu em uma ou ambas as modalidades de forma sistemática ou assistemática (Anexo 2)

A coleta foi realizada no período de aula, em sala cedida pela escola. O local apresentava ventilação e iluminação adequadas, sem interferência de ruídos. As crianças se posicionaram em frente à examinadora, sentadas em cadeira confortável, com o tronco mantido verticalmente ao plano horizontal e a cabeça orientada com o plano de Frankfurt e o plano sagital mediano perpendicular no plano horizontal. Os procedimentos foram realizados com as devidas medidas de biossegurança.

Para análise e avaliação, considerou-se como ceceio interdental anterior o avanço ou projeção da língua, entre os dentes incisivos superiores e inferiores no fonema /s/ e como ceceio lateral quando a interposição da língua ocorria nos demais dentes excetuando-se os incisivos ou quando os dentes anteriores levados numa relação topo-a-topo, os pré-molares separados demasiadamente e havendo escape lateral do fluxo de ar no fonema /s/.

Foi realizada a oroscopia, com auxílio de espátula de madeira, para observação das condições anatômicas e o tipo de oclusão e mordida, tendo em vista que alterações nas relações espaciais entre as extremidades incisivais inferiores e as superfícies dos dentes antagonistas podem gerar distorções sonoras nos fonemas fricativos. Dessa forma, considerou-se como mordida aberta anterior o distanciamento vertical entre as bordas dos incisivos; para mordida cruzada a inversão da oclusão em que os dentes superiores ocluem internamente aos seus opostos inferiores e sobremordida, quando a oclusão dos incisivos superiores encobria os inferiores além de um terço.

Para complementar a oroscopia investigaram-se a presença de sinais típicos de respiração oral, tais como: características faciais, lábios entreabertos, hipotonia labial, língua no assoalho da boca no repouso, que poderiam ser indicativos do uso de respiração oral ou oronasal. Considerou-se positivo a ocorrência de no mínimo dois sinais, dois quais seriam obrigatórios a ausência de selamento dos lábios e língua baixa no repouso. A presença de tais sinais acompanhados da afirmação dos pais quanto à manutenção da boca aberta foi, no presente estudo, considerada e denominada de sinais sugestivos de respiração oral (SSRO). 
Tabela 1. Distribuição da amostra conforme presença e ausência de ceceio e os fatores de risco investigados

\begin{tabular}{|c|c|c|c|c|c|c|}
\hline \multirow[t]{2}{*}{ Fatores de risco } & \multicolumn{2}{|c|}{ Com ceceio } & \multicolumn{2}{|c|}{ Sem ceceio } & \multirow[t]{2}{*}{$x^{2}$} & \multirow[t]{2}{*}{ Valor de $\mathrm{p}$} \\
\hline & $\mathrm{n}$ & $\%$ & $\mathrm{n}$ & $\%$ & & \\
\hline Sinais sugestivos de respiração oral & 19 & 9,5 & 52 & 26 & 4,307 & 0,037 \\
\hline Chupeta & 29 & 14,5 & 106 & 53 & 1,662 & 0,197 \\
\hline Sucção dedo & 3 & 1,5 & 12 & 6 & 0,010 & 0,918 \\
\hline Mamadeira & 8 & 4 & 14 & 7 & 4,842 & 0,027 \\
\hline Alterações praxias & 32 & 16 & 95 & 47 & 9,054 & 0,002 \\
\hline Alterações oclusão & 35 & 17,5 & 93 & 46,5 & 0,626 & 0,0001 \\
\hline Base & \multicolumn{2}{|c|}{38} & \multicolumn{2}{|c|}{162} & & \\
\hline
\end{tabular}

Para análise dos resultados, utilizou-se o programa Statistical Analisys System (SAS) versão 8.02, além da estatística não-paramétrica, adotando o Teste Qui-quadrado, para verificar se duas variáveis qualitativas e seus níveis possuíam ou não dependência (associação) estatística. Foram considerados estatisticamente significantes os valores com $\mathrm{p}<0,05$.

Todos os responsáveis pelos alunos assinaram o Termo de Consentimento Livre e Esclarecido, ficaram cientes dos propósitos desta pesquisa e foram assegurados quanto a sua privacidade.

\section{RESULTADOS}

Verificou-se a presença de ceceio em 19\% da amostra, sendo que em $14 \%$ observou-se o ceceio anterior, em 3,5\% o ceceio lateral e em $1,5 \%$ ambos desvios do tipo ceceio anterior e lateral.

Cabe ressaltar que durante o protocolo de avaliação houve diferença na produção do fonema /s/, pois $4 \%$ da amostra realizaram a nomeação de figuras e a repetição de frases de forma correta, porém durante a linguagem espontânea apresentaram ceceio anterior.

O número de crianças com ceceio anterior diminuiu com o aumento da idade, o que não aconteceu com o ceceio lateral:

- oito anos: dez alunos com ceceio anterior e um com ceceio lateral;

- nove anos: sete alunos com ceceio anterior e dois com ceceio lateral, sendo um aluno com ambos os desvios;

- dez anos: cinco alunos com ceceio anterior, dois com ceceio lateral e dois com ambas as alterações;

- 11 anos: seis alunos com ceceio anterior e dois com ceceio lateral.

Encontrou-se ceceio em $26 \%$ de crianças do sexo feminino e em $12 \%$ do sexo masculino.

Na Tabela 1 observa-se que nesta amostra há fatores de risco associados ao ceceio.

\section{DISCUSSÃO}

Este estudo encontrou a frequência de ceceio em $19 \%$ da amostra. Considera-se que a produção do fonema /s/ requer condições precisas de posicionamento e forma das estruturas orais $^{(2-4)}$ assim como está diretamente relacionada à maturação do sistema estomatognático e às funções neurovegetativas associadas: respiração, sucção, mastigação e deglutição ${ }^{(15)}$. Por esta razão, é tão frequentemente pronunciada incorretamente. Este resultado é semelhante ao encontrado no estudo que verificou a incidência de ceceio em 68 crianças com idade entre três e dez anos, na Casa de Ação Social Cristo Rei, residentes na Grande Vitória/ES ${ }^{(14)}$. É, porém, menor que a frequência encontrada em estudo com amostra de crianças de menos de sete $\operatorname{anos}^{(3,8)}$. Tal achado reforça a hipótese de redução da distorção fonêmica com o aumento da idade.

Mais ainda, quando se analisa a presença de ceceio nas diferentes faixas etárias nesta amostra, observa-se que o ceceio anterior diminui com a idade e estabilizou-se em torno dos dez anos. Tal resultado é semelhante ao estudo com 50 crianças de mesma faixa etária realizado em escola particular ${ }^{(12)}$. É importante ressaltar que aos 11 anos de idade dá-se o refinamento da coordenação têmporo-espacial de componentes individuais do mecanismo de produção de fala, época em que um controle de movimentos articulatórios mais preciso é atingidoe o controle motor de fala se assemelha ao padrão adulto ${ }^{(16)}$.

Por outro lado, não se percebe o declínio na frequência do ceceio lateral com a idade; ao contrário, na amostra pesquisada, encontrou-se um ligeiro aumento. Como foi baixa a frequência do ceceio lateral no presente estudo, sugerem-se outras investigações dessa distorção nas diferentes faixas etárias. De qualquer forma, tal dado reforçaria a necessidade de atendimento fonoaudiológico especializado ao ser diagnosticado ceceio lateral, diferentemente do anterior, quando é possível muitas vezes apenas acompanhar a evolução, conforme o crescimento e desenvolvimento orofacial.

Ressalta-se a importância da linguagem espontânea na avaliação e intervenção terapêutica para a queixa de ceceio, já que houve diferença de $4 \%$ entre a produção espontânea e a eliciada por meio de figuras ou repetição.

De acordo com a distribuição da amostra por sexo é notável a diferença de ceceio do sexo feminino (26\%) sobre o masculino (12\%). Tal achado difere dos resultados de estudos anteriores que não encontraram diferenças quanto ao gênero ${ }^{(3,9,11)} \mathrm{ou}$ inclusive encontraram maior frequência no sexo masculino ${ }^{(4)}$. Evidencia-se a necessidade de pesquisas que visem verificar a ocorrência de ceceio relacionando-o a esta variável.

Os resultados do presente estudo demonstraram que o ceceio apresenta como fatores de risco os SSRO ( $\mathrm{p}=0,037)$, o hábito prolongado de mamadeira ( $\mathrm{p}=0,027)$, a alteração na realização das praxias de língua $(\mathrm{p}=0,002)$ e as alterações de mordida $(\mathrm{p}=0,0001)$.

A respiração oral favorece a ocorrência de ceceio anterior 
ou lateral ${ }^{(17)}$. Quando se adquire o hábito de ficar com a boca entreaberta, a língua adquire uma posição mais baixa. Como consequência desse posicionamento inadequado de lábios e língua, pode ocorrer flacidez da língua e dos lábios, principalmente do inferior, retração de lábio superior e possíveis alterações oclusais, sendo as mais comuns as mordidas abertas e cruzadas ${ }^{(16)}$. Também foi alta a frequência de ceceio anterior (30\%) e de ceceio lateral (10\%) em um estudo de 22 crianças de ambos os sexos, com diagnóstico de respiração oral entre quatro e $11 \operatorname{anos}^{(13)}$. Na presença de sinais sugestivos de respiração oral é, portanto, recomendável complementar a avaliação fonoaudiológica com a avaliação otorrinolaringológica.

Pode-se comparar o alto índice de hábito prolongado da mamadeira neste estudo, com a pesquisa ${ }^{(18)}$ que investigou a associação entre sucção, condições miofuncionais orais e fala, realizada com 50 crianças pré-escolares com seis e sete anos, na qual concluiu-se que o distúrbio de fala foi associado à maior duração do aleitamento artificial e da sucção não-nutritiva. $\mathrm{O}$ estudo refere que o prolongamento da sucção pode modificar o ambiente oral, dentre outras coisas, provocando distúrbios miofuncionais orais e, consequentemente, dificultando os ajustes motores finos necessários para a articulação da fala.

Problemas das estruturas orofaciais que surgem em decorrência de hábitos parafuncionais, como o uso prolongado da mamadeira, podem provocar alteração na relação de dentes superiores e inferiores e hiperatividade da musculatura elevadora da mandíbula, ocasionando prejuízo na fala ${ }^{(16)}$.

Assim como na presente pesquisa, o estudo que verificou a ocorrência de hábitos orais viciosos e distúrbios fonoarticulatórios em indivíduos com deglutição atípica ${ }^{(12)}$, a maioria da amostra apresentou hábitos orais viciosos, cuja maior ocorrência foi a sucção de mamadeira.

Por outro lado, não houve associação de ceceio com hábitos de sucção não nutritiva (dedo e chupeta). Diferente dos resultados da pesquisa realizada em escolas particulares das cidades de Ipatinga, Resende Costa e Belo Horizonte/ $\mathrm{MG}^{(11)}$, nos quais verificou-se que uma criança com sucção não nutritiva tem, aproximadamente, quatro vezes mais chance de ser uma criança com ceceio na fala do que uma criança sem esses hábitos.

Dentre os fatores de risco para a ocorrência de ceceio nesta pesquisa, encontra-se a alteração de oclusão de mordida aberta. As relações espaciais entre as extremidades incisais inferiores e as superfícies dos dentes antagonistas, são fatores determinantes na produção de vários fonemas fricativos. Assim como a relação destes com a língua e os lábios. Alterações nessas relações podem gerar várias distorções sonoras como o ceceio $^{(16)}$.

Os resultados quanto à oclusão do presente estudo concordam com algumas pesquisas que investigaram essa associação ${ }^{(19)}$. Foi importante a frequência $(50 \%)$ de ceceio encontrada, em pesquisa com 50 crianças entre cinco e 14 anos com mordida aberta anterior ${ }^{(10)}$. Tais achados reforçam a necessidade da avaliação do sistema estomatognático, nos casos de distorção na fala ${ }^{(19)}$.

Por outro lado, vários estudos ${ }^{(4,8-9,12)}$ sugerem as alterações da oclusão apenas como facilitadores da distorção fonêmica, sem serem única e totalmente justificadas por esta alteração. Porém, o tipo de mordida é a má oclusão que mais se associa ao ceceio $^{(12,20)}$. A mordida aberta anterior pode levar a adaptações no posicionamento da língua, interpondo-a, protruíndo-a ou elevando-a ${ }^{(21-22)}$. A mordida aberta lateral e a cruzada também podem produzir distorção do fonema ${ }^{(3,12)}$.

A alteração na realização de praxias de língua foi um achado importante nos alunos que apresentaram ceceio. Constatouse a não realização de movimentos com a língua, em especial, para realizar canolamento. A partir desse achado sugere-se a realização de estudos que verifiquem a relação entre ceceio e praxias de língua, em especial de canolamento lingual. $\mathrm{O}$ fonema /s/ é produzido elevando-se as laterais da língua contra o palato duro, impedindo o escape lateral do $\operatorname{ar}^{(3)}$ e esta habilidade também é necessária para a realização da praxia.

Estudo $^{(19)}$ que investigou os achados fonoaudiológicos em crianças de quatro a seis anos com queixa de distúrbio de fala, obteve resultados semelhantes aos achados neste estudo, pois verificou diminuição da mobilidade da língua e também dos lábios e/ou bochechas.

Outro estudo que comparou a produção de fricativas sibilantes (s, z) e dentais ( $\mathrm{f}, \mathrm{v})^{(23)}$ encontrou que as fricativas alveolares (s, z) apresentam pontos articulatórios mais estáveis, ou seja, não permitem várias trajetórias articulatórias para atingir o mesmo alvo acústico. Esse achado reforça a afirmação da extrema precisão na produção articulatória do fonema /s/ e elucida também o fato de desvios de tal consoante ser tão frequente, pois pequenas variações no formato e localização de língua alteram a produção final.

\section{CONCLUSÃo}

Em razão do que foi descrito nesta pesquisa, pode-se concluir que foi importante a ocorrência de ceceio em 19\% dos 200 alunos avaliados, da rede escolar municipal de Itaqui/RS.

Quanto à análise por faixa etária, evidencia-se a diminuição e a estabilização do ceceio anterior conforme a idade aumenta, o que não acontece com o ceceio lateral. Além disso, houve predomínio do sexo feminino, o que contradiz muitas pesquisas de distúrbio articulatório. A partir deste resultado, novas pesquisas poderão analisar a ocorrência de ceceio conforme o sexo.

Ressaltam-se ainda, nesta pesquisa que sinais sugestivos de respiração oral, o hábito prolongado da mamadeira, as alterações de praxias de língua e as alterações oclusais, foram fatores de risco estatisticamente significantes para a ocorrência de ceceio. 


\begin{abstract}
Purpose: To verify the occurrence of lisp in eight- to 11-year-old children from public schools in Itaqui/RS (Brazil), according to gender and age group, and to associate the lisp with possible risk factors, such as sucking habits (pacifier, finger and bottle) up to six years of age, malocclusion, oral breathing and tongue praxis. Methods: An observational, exploratory, contemporary, prospective, group study was conducted using a questionnaire applied to caregivers and a speech-language evaluation that included examination of oral structures, tongue praxis, signs suggestive of oral breathing, and speech sounds articulation. Non-parametric statistics (Chisquare) was used in order to verify the association between variables. Results: Two hundred children with ages ranging from eight to 11 years took part in the study (50\% male and 50\% female). The occurrence rate of lisp was 19\%, higher for female subjects. It was observed that anterior lisp decreases with age, but the same did not occur for lateral lisp. Suggestive signs of oral breathing $(\mathrm{p}=0.037)$, prolonged use of bottle $(\mathrm{p}=0.027)$, tongue praxis alterations $(\mathrm{p}=0.002)$, and malocclusion $(\mathrm{p}=0.0001)$ were the risk factors that showed statistical association. Conclusion: The occurrence of lisp was high, and affected more female subjects. Most of the risk factors were significantly associated.
\end{abstract}

Keywords: Speech-language pathology; Articulation disorders; Sucking behavior; Dental occlusion; Mouth breathing

\title{
REFERÊNCIAS
}

1. Gierut JA. Treatment efficacy: functional phonological disorders in children. J Speech Lang Hear Res. 1998;41(1):S85-100.

2. Chen H, Stevens KN. An acoustical study of the fricative /s/ in the speech of individuals with dysarthria. J Speech Lang Hear Res. 2001;44(6),1300-14.

3. Fonseca RA, Tucci TA, Rodriguez RCL, Gomes ICD, Bianchini EMG. A correlação entre ceceio frontal e o crescimento infantil. Rev Soc Bras Fonoaudiol. 2005;10(4):211-7.

4. Tomé MC, Farias SR, Araújo SM, Schimitt BE. Ceceio interdental e alterações oclusais em crianças de 03 a 06 anos. Pró-Fono. 2004;16(1):19-30.

5. Oliveira JTN, Oliveira ZSB. Desvio fonético $X$ desvio fonológico: algumas considerações. J Bras Fonoaudiol. 2004;5(20):172-6.

6. Sociedade Brasileira de Fonoaudiologia - SBFA. Comitê de Motricidade Oral (MO) [Internet]. Documento Oficial 03/2003 [cited 2005 Dez 08]. Disponível em: http://www.sbfa.org.br

7. Subtelny JD, Mestre JC, Subtelny JD. Comparative study of normal defective articulation of /s/ as related to malocclusion and deglutition. J Speech Hear Disord. 1964;29(3):269-85.

8. Moura AL. O ceceio anterior em crianças de 3 a 7 anos. In: Marchesan IQ, organizadora. Tópicos em fonoaudiologia. São Paulo: Lovise; 1994. p. 231-8.

9. Junqueira $P$, Guilherme A. ocorrência de sigmatismo interdental em crianças de três à oito anos e sua relação com idade e oclusão dental. In: Marchesan IQ, Zorzi JL, Gomes ICD, organizadores. Tópicos em fonoaudiologia 1996. São Paulo: Lovise; 1996. III. p. 275-87.

10. Peña NVR, Cabrera NR, Mozo BC; Martínez RS. Anomalias da oclusão e transtornos na articulação das palavras. J Bras Fonoaudiol. 2004;5(20):155-9.

11. Frias JS, Forasti FNR, Carmona AS, Di Ninno CQMS. Relação entre ceceio anterior e crescimento craniofacial e hábitos de sucção não nutritiva em crianças de 3 a 7 anos. Rev CEFAC. 2004;6(2):177-83.
12. Pereira MMB, Bianchini EBG, Carvalho GGT, Jardim ZMG. Investigação da ocorrência e caracterização de distorções do /s/ em crianças de 3 a 10 anos. Rev Soc Bras Fonoaudiol. 2003;8(1):10-7.

13. Bicalho GP, Motta AR, Vicente LCC. Avaliação da deglutição em crianças respiradoras orais. Rev CEFAC. 2006;8(1):50-5.

14. Machado AS. Verificação da ocorrência de ceceio em crianças entre 3 e 10 anos. [Dissertação]. Rio de Janeiro: Universidade Veiga de Almeida; 2006.

15. Tanigute CC. Desenvolvimento das funções estomatognáticas. In: Marchesan IQ. Fundamentos em fonoaudiologia: aspectos clínicos da motricidade oral. Rio de Janeiro: Guanabara Koogan; 1998. p. 1-6.

16. Felício CM. Fonoaudiologia aplicada a casos odontológicos: motricidade oral e audiologia. São Paulo: Pancast; 1999. p. 51-64.

17. Marchesan IQ. Alterações músculo-esqueletais. In: Ferreira LP, BefiLopes DM, Limongi SCO, organizadores. Tratado de fonoaudiologia. São Paulo: Roca; 2004. p 294-5.

18. Felício CM, Ferreira-Jeronymo RR, Ferriolli BHVM, Freitas RLRG. Análise da associação entre sucção, condições miofuncionais orais e fala. Pró-Fono. 2003;15(1):31-40.

19. Franco DP, Ávila CRB. Achados fonoaudiológicos de crianças com queixa de distúrbio de fala. Pró-Fono. 2000;12(1):40-7.

20. Leite AF, Silva SB, Britto ATBO, Di Ninno CQMS. Caracterização do ceceio em pacientes de um Centro Clínico de Fonoaudiologia. Rev Soc Bras Fonoaudiol. 2008;13(1):30-6

21. Souza RF, Compagnoni MA. Relation between speaking space of the /s/ sound and freeway space in dentate and edentate subjects. Braz Oral Res. 2004;18(4):333-7.

22. Maciel CTV, Leite ICG. Aspectos etiológicos da mordida aberta anterior e suas implicações nas funções orofaciais. Pró-Fono. 2005;17(3):293-302.

23. Tabain M. Variability in fricative production and spectra: implications for the hyper- and hypo- and quantal theories of speech production. Lang Speech. 2001;44(Pt 1):57-94. 
Anexo 1. Questionário aos pais

Nome da Criança:

Data Nascimento:

Idade

1 - Seu filho fez terapia fonoaudiológica?

2 - Seu filho usa ou usou aparelho ortodôntico?

3 - Seu filho chupou chupeta?

4 - Caso a resposta for sim, até quantos anos chupou?

5 - Seu filho chupa o dedo?

6 - Caso a resposta for NÂO, até quantos anos chupou

$$
\begin{array}{ll}
\text { Sim } & \text { Não } \\
(\quad) & (\text { ) } \\
(\quad) & (\text { ) }
\end{array}
$$

7- Caso a resposta for sim, durante quanto tempo ele chupa o dedo?

$\begin{array}{ll}\text { O dia todo } & (\text { ) } \\ \text { Durante o dia e para dormir } & (\text { ) } \\ \text { Somente para dormir } & (\text { ) }\end{array}$

8 - Seu filho toma mamadeira?

$$
\text { Sim ( ) Não ( ) }
$$

9 - Caso a resposta for SIM, quantas mamadeiras ele toma diariamente?

10 - Seu filho fica com a boca aberta ou entreaberta?

$$
\text { Sim ( ) Não ( ) }
$$

11 - Caso a resposta for SIM, durante quanto tempo ele fica com a boca aberta ou entreaberta?

$$
\begin{array}{ll}
\text { O dia todo } & (\text { ) } \\
\text { Durante o dia e para dormir } & (\text { ) } \\
\text { Somente para dormir } & (\text { ) }
\end{array}
$$

12- Seu filho tem problemas respiratórios?

$\begin{array}{ll}\text { Rinite } & (\text { ) } \\ \text { Bronquite } & (\text { ) } \\ \text { Asma } & (\text { ) } \\ \text { Resfriado frequentes } & (\text { ) }\end{array}$

Anexo 2. Protocolo de avaliação fonoaudiológica

Nome:

Escolaridade:

Nomeação de Figuras:

\section{Sapato}

Bolsa

Cenoura

Travesseiro

Cir

Piscina

Sorvete

Palhaço

Super-homem

Açucareir

Repetição de frases:

O Sapo pulou na moça.

Selma pintou compincel.

A polícia tocou a sirete.

Sônia assoprou a vela.

Sueli ficou assustada.

Praxias língua: Sequência

Fora/dentro:

Estalar:

Afinar/alargar:

Canola ponta:

$($ )
$($ )
$($ )

D.N.:

Sexo:

Idade:

$\begin{array}{cc}\begin{array}{c}\text { Ceceio lateral } \\ (\text { ) }\end{array} & \text { Ceceio anterior } \\ (\text { ) } & (\text { ) } \\ (\text { ) } & (\text { ) } \\ (\text { ) } & (\text { ) } \\ (\text { ) } & (\text { ) } \\ (\text { ) } & (\text { ) } \\ (\text { ) } & (\text { ) } \\ (\text { ) } & (\text { ) } \\ (\text { ) } & (\text { ) } \\ (\text { ) } & (\text { ) }\end{array}$

Ceceio lateral Ceceio anterior

$\begin{array}{ll}() & (\text { ) } \\ () & (1) \\ () & (\text { ) } \\ () & (\text { ) }\end{array}$


Observação fala espontânea:

Sinais típicos de respiração oral:

Oroscopia:

$\begin{array}{llllll}\text { ( ) Mordida aberta } & \text { ( ) anterior } & \text { ( ) lateral } & & \\ \text { ( ) Mordida cruzada } & \text { ( ) lateral } & \text { ( ) D } & \text { ( ) E } & \text { ( ) bilateral } & \text { ( ) anterior } \\ \text { ( ) Palato ogival } & \text { ( ) palato plano } & & & & \\ \text { ( ) Diastemas } & & & & \end{array}$

( ) Diastemas

( ) Sobremordida 